\title{
Poder y Ciudadanía. Estudios sobre Hobbes, Foucault, Habermas y Arendt Maximiliano Figueroa (ed.)
}

\author{
UAI-RIL editores, Santiago de Chile, 2014, 152 páginas.
}

\author{
Felipe Torres N. \\ Universidad Diego Portales, Santiago, Chile. \\ Email: felipe.torres@udp.cl
}

Lo primero que cabría señalar es que esta presentación se centrará en las palabras que convoca el título del libro y que, por supuesto, pueden verse en diferentes grados problematizadas al interior de los textos aquí presentados.

Inicialmente se podría estar tentado a decir que la dicotomía poder y ciudadanía ya no puede pensarse de manera separada, en la medida que los sucesivos ejemplos de movimientos ciudadanos dan cuenta de la relación intrínseca entre la ciudadanía y su poder, o empoderamiento.

Un título como "poder y ciudadanía” pareciera no suscitar muchas dudas respecto de qué sea aquello que tratará su contenido. Es un título claro, en el que se presenta la unión de las palabras "poder” y “ciudadanía”, donde lo que se nombra son dos temas: el poder por un lado y la ciudadanía por otro. Pero esa unión establecida por la conjunción “y” puede tener más de un sentido, por lo menos dos.

a) por un lado encontramos “poder y ciudadanía” como la unión de dos ámbitos separados en el que "poder" supondría una dimensión escindida de "ciudadanía";

b) mientras que de otro lado podemos remitirnos a poder y ciudadanía como dos dominios de una misma nomenclatura.

En el primer caso puede que la " $y$ ” no se limite simplemente a juntar lo que ya de algún modo se hallaría unido en la realidad, sino que se encargue de la nada fácil tarea de juntar cosas absolutamente distintas. En este caso la "y” no haría más que permitir la consideración simultánea de dos términos que señalan ámbitos sin posibilidad de intersección. Si el escrito que se está titulando va a tratar justamente de hacer explícita esa imposibilidad de intersección, si el intento consistirá en distinguir lo mejor posible entre los dos terrenos nombrados en el título, entonces la función de la "y" que se halla en medio de ambos términos es permitir sólo la consideración conjunta de ellos, consideración sin la cual sería imposible la posterior 
distinción. En este caso la “y”, como conjunción que es, poder.er o la ciudadan versal cumple también la función de unir, pero logra la más débil unión imaginable: sólo la unión indispensable previa a una radical distinción.

Pero puede que esta " $y$ ” cumpla una labor distinta. Puede que la "y” indique una cercanía fundamental entre los dos términos que une, cercanía que obligue a pensar a ambos como reunidos por un mismo ámbito, como envueltos por un sentido compartido o como pertenecientes al mismo lugar. En este caso la "y" cobra una importancia tal que logra remitir a un tema que no es indicado por ninguno de los términos, sino eso que los envuelve y permite que la unidad entre ambos sea posible. Es lo que sucedería con títulos como "azul y negro", "pintura y escultura”, "tigres y leones”, o cualquier otro en el cual salte a la vista un tema que sobrepasa a cada una de las palabras y que resulta sugerido por la proximidad entre ellas y por la labor de unión de la "y". En este caso la "y" repite una unión dada de antemano con lo cual alcanza a sugerir un tema mayor en el que quedan envueltos los dos términos.

Lo curioso es que la "y" de "poder y ciudadanía” no parece decidirse de buenas a primeras por una de las dos posibilidades que acabamos de presentar. Basta una rápida ojeada al texto cuyo título nos está aquí ocupando para darse cuenta que poder y ciudadanía no siempre remiten fácilmente a un ámbito común, que no son compañeros fáciles de una misma residencia.

Lo cierto es que da la impresión que más bien el libro pone grandes esfuerzos en establecer distinciones oportunas entre poder y ciudadanía, donde ciudadanía se muestra justamente como una propuesta que consistiría en escapar al dominio del poder entendido como control.

Sin embargo, el título parece querer indicar también algo así como un ámbito común desde el cual serían posibles tanto el poder como la ciudadanía. La ligazón dada por la "y” no sería, entonces, tan sólo la ligazón necesaria para una radical distinción, no se remitiría a la mera consideración conjunta en la mente de quien las piensa, sino que se trataría de una ligazón fundada en el modo de aparecer "poder” y “ciudadanía” en la realidad. Pero si poder y ciudadanía se encontraban ya unidas, independientemente de la consideración del analista, ¿̇a qué tanto afán por establecer una radical distinción entre ambas? Antes de intentar una respuesta habría que fijarse en las afirmaciones que se encuentran al interior de esta pregunta, una de las cuales establecería una radical distinción entre poder y ciudadanía. La pregunta, entonces, previa a la que recién formuláramos, se vuelve otra: ¿qué tan radical es la distinción propuesta entre poder y ciudadanía?

Naturalmente urgar en esta relación de manera satisfactoria excede los propósitos de esta presentación. Sin embargo y a la luz de ciertos planteamientos presentes en los textos que componen este libro, resulta posible esbozar ciertas ideas relevantes en función de aclarar los vínculos. 
Inicialmente podemos encontrar una formulación que expone a la ciudadanía como correlato del poder en el momento que éste siempre es resultado de la agregación de muchos. Es así como en "Hannah Arendt y el sentido de lo político” (pp. 123-152) Maximiliano Figueroa, siguiendo a la autora en ¿Qué es la Política? (1997b), plantea que el poder nunca es patrimonio de un individuo, sino de una congregación y en la medida que ésta existe. Del mismo modo que una manifestación del poder, como es la ley, tampoco escapa al hecho de estar permanentemente radicada en la determinación de un pueblo. Así, nos encontramos en un primer momento frente a la imposibilidad de separar ambas esferas sin por ello confundirlas, o quizás confundiéndolas lo suficiente para mostrar la familiaridad entre ambas.

Para tener mayor claridad sobre esto último conviene revisar otro planteamiento, esta vez en el texto de Pablo Salvat titulado "Habermas y la democracia deliberativa como democracia radical” (pp. 93-122), quien siguiendo a al filósofo alemán recuerda la importante hipótesis de la colonización del mundo de la vida por parte de lógicas sistémicas provenientes de dos grandes ámbitos (político y económico) que han logrado un nivel de autonomía tal que impide la identificación sin más entre el poder y su origen. Puesto que la descripción de sociedades de capitalismo avanzado afirma que éstas requieren una cierta escisión entre dinámicas de dinero y poder que se desenvuelvan de manera autónoma, es difícil observar cómo el poder es, hoy en día, resultado de la ciudadanía. Esto no pone en duda la certeza del argumento antes visto de Arendt, sino más bien enfatiza el problema que aquejaría a la creencia de una acción eficaz en el espacio público sin considerar aspectos sistémicos que escapan a la actividad concertada de una multitud. El poder es de la ciudadanía también en Habermas, como también lo es diagnosticar los obstáculos que este ejercicio enfrenta permanentemente en la actualidad.

Otro problema es la manera en que se puede articular la relación entre poder y ciudadanía en el sentido de cómo el poder adquiere formas históricas precisas. En este sentido es que toma fuerza la idea de una imagen como la de un soberano, ejemplificada a través de la figura de un Estado. Es así como Diego Rossello en "Hobbes y el hombre lobo: devenir animal en (y ante) la soberanía” (pp.11-35) nos muestra que el soberano de Hobbes es la superación de una animalidad (la animalidad del hombre como lobo del hombre) que recurre a la figura de otra animalidad, el Leviatán, para suprimir precisamente la primera bestialidad del estado de naturaleza. Es, por tanto, la articulación de una fuerza no humana, o más allá de lo humano, lo que funda la estabilización de un poder colectivo en la figura de un Estado.

De otra parte el poder tampoco puede identificarse de manera directa con la acción de un soberano investido, puesto que su misma constitución excede cualquier tipo de formalidad. Es así como el poder se descompone o ramifica en distintas versiones. Muchas de las cuales permean dimensiones de vida que no pueden identificarse sin crítica con un centro. Omar Aguilar en "El poder de la sociedad: una lectura sociológica de Michel Foucault" 
(pp. 37-91) nos recuerda cómo, entre otras cosas y siguiendo al pensador francés, el poder no es una posesión y por lo mismo sus espacios de ejecución tampoco pueden reducirse al lugar de las decisiones reconocibles. Es así como aparatos de producción, instituciones, familias, relaciones de conocimiento, económicas o sexuales, son dominios del poder. Una cosa importante en todo este escenario es que este poder proviene precisamente de los mismos sitios sociales donde este tiene lugar. Esto no niega el hecho de que existan, digámoslo así, estructuras sociales que acompañan estas ejecuciones de poder, sino únicamente que no hay que buscar la fuente del poder desde arriba, verticalmente, como constriñendo desde fuera, sino como, citando a Aguilar, relaciones que son al mismo tiempo "intencionales y no subjetivas” (p. 84).

Esto último, a mi juicio, tiene importantes consecuencias para la noción de ciudadanía ya que ésta no podría, y volviendo a la pregunta inicial por la "y" del título de este libro, concebirse separada de la formación del poder. Ni la ciudadanía es ajena a la formación del poder desde arriba, ni tampoco está más allá de las formas, perversiones, límites y posibilidades que este supone. La ciudadanía es también una forma del ejercicio del poder, y viceversa, el poder un ejercicio ciudadano.

En este punto se vuelve interesante considerar que si la ciudadanía aloja las mismas dimensiones del poder distinguibles más allá de ella, es también un campo en el que se presentan los desafíos del ejercicio del poder. Si esto es así no es descabello preguntar por la actuación o performance de la ciudadanía, no al modo de un sujeto colectivo, sino más bien a la manera de un encuentro de subjetividades. Así es como se podría aventurar que otros autores conciben la acción política de la ciudadanía bajo otros epítetos: Multitud en Spinoza y luego Antonio Negri y Paolo Virno, o Pueblo en una vasta tradición que encuentra en Hobbes y Rousseau destacados exponentes. Si bien sabemos que ambos conceptos remiten a significados distintos, en términos generales, y en cada autor tomado individualmente, es posible rescatar un elemento común tanto a pueblo y multitud como, incluso ciudadanía, en la medida que todos remiten al espacio de lo público.

Ahora bien ¿es la noción de ciudadanía el mejor término para definir políticamente un espacio vital de ejecución de poder? Es habitual escuchar hablar de ciudadanía empoderada. Este argumento se escucha apelando a lo deseable que sería tener mayores atribuciones por parte de quienes se ven directamente involucrados en distintas situaciones y en relación a la toma de decisiones que afectan diferentes ámbitos: fundamentalmente desde su entorno más directo, hasta en menor medida los cursos generales de la sociedad. Pero este empoderamiento no acaba ahí o no puede hacerlo, ya que la ciudadanía requiere ir más allá de las atribuciones muchas veces legales que supone el ser ciudadano o ciudadana. Aquí es cuando el concepto se ve limitado por lo mismo que pretende, a saber, empoderamiento ciudadano que golpea con los criterios de la soberanía, por ejemplo del Estado, y todo lo que ello implica. Aquí es cuando aventuramos sin aden- 
trar en ello, que un concepto como el de multitud podría resultar oportuno en la medida que supone temporalidades y encuentros aleatorios, que no pueden circunscribirse a la normatividad presente sino para abrir o hacer visibles otras normatividades o que, como diría Paolo Virno en Gramática de la multitud "... es la forma de existencia social y política de los muchos en tanto muchos” (2003: 22).

Para terminar y recapitulando la pregunta con que comenzamos esta presentación, todo indica que la "y" que vincula poder y ciudadanía es la forma de presentar dos ámbitos de una misma dimensión. No se encuentran en dominios distintos, sino más bien aparejados, incluso a ratos de manera indistinguible. La "y" de poder y ciudadanía es también la "y" de poder y pueblo, poder y soberanía, o más sucintamente, poder y política.

Tomando en consideración esto el libro presente es una conveniente aproximación a algunos de los problemas que surgen una vez que poder y ciudadanía son pensados como fenómenos que se interpelan mutuamente y que resultan de tanta pertinencia para la actualidad política tanto a nivel nacional como global. 
Polis, Revista Latinoamericana, Volumen 13, $N^{\circ}$ 38, 2014

\section{Bibliografía}

Arendt, Hannah (1997), ¿Qué es la Política? Paidós, Barcelona.

Virno, Paolo (2003), Gramática de la Multitud. Traficantes de Sueños, Madrid. 